\title{
Auf der Couch
}

Wie geht's? Nein, das fragen Sie nicht, Herr Doktor, ich weiss, und das finde ich gut. Ich musste mich zuerst daran gewöhnen, ich meine, akzeptieren, dass ich losplappern kann, ohne dass Sie mir vorher ein paar höfliche Fragen stellen. Und jetzt gefällt es mir, nur kein Smalltalk, das hasse ich; stellenweise komme ich mir als Aussenseiterin vor. Die meisten tun es, doch, doch; wobei: Genau betrachtet ... ich mache es eigentlich auch, selten allerdings. Wenn mich aber jemand lange mit Smalltalk terrorisiert, verdufte ich lieber.

Also: Auf der Abteilung nichts Neues. In der letzten Woche gab es keine grossen Probleme, Gott sei Dank, von der Arbeit werde ich also nicht reden ... es sei denn, es kommt mir etwas Wichtiges in den Sinn. Lieber gleich von ... tja, wovon eigentlich? Ist ja egal! Sagen wir ... von Philipp. Bitte? Ach nein, Träume hatte ich diesmal keine, diesen Punkt können wir gleich abhaken. Schade! Ich weiss, heute werde ich Sie enttäuschen, Herr Doktor, es tut mir leid. Einen Schreibblock und Bleistift habe ich auf dem Nachttisch, wie Sie es mir seinerzeit empfohlen haben.

Philipp also. Vorgestern angerufen. Schön! Fast zwei Wochen hatte ich auf ein Lebenszeichen von ihm gewartet. Noch drei weitere Tage, dachte ich, und dann meldest du dich selber; ungern, klar, aber die ewige Warterei ist auch nicht so toll. Es war allerdings nicht nötig, er rief an; ich gab mich überrascht, cool, leicht distanziert ... vielleicht übertrieb ich ein wenig, kann sein. Er sprach plötzlich am Telefon viel leiser, fragte, ob er störe, ob er sich nicht lieber später melden solle. Ach nein, sagte ich, schon gut, wir können ein bisschen plaudern, wenn ihm danach sei.

Es war keine lange Unterhaltung, wir verabredeten uns ziemlich rasch. Nicht weit von hier, von Ihrer Praxis, Herr Doktor, im Restaurant «Mondial». In dieser Gegend kenne ich mich gut aus ... meine Güte, das sind bald zwei Jahre, seit ich regelmässig zu Ihnen komme, tja, so schnell vergeht die Zeit!

Philipp gegenüber bin ich nach wie vor ambivalent; ein gutaussehender Mann, das auf jeden Fall, durchaus sympathisch, aber irgendwie - schwer zu beschreiben - auf Anhieb nicht unbedingt mein Typ. Nur keine Eile, sagte ich mir, alles kann sich noch entwickeln; auf dieses Date, das dritte in der Reihe, freust du dich doch. Wie komme ich aber hin?, fragte ich mich. Diesmal nicht mit dem Auto, denn etwas trinken werden wir bestimmt. Zu Fuss? Na ja. Ziemlich weit, erstens, und zweitens, verschwitzt will ich mich nicht unbedingt im «Mondial» umarmen lassen; aus diesem Grund bleibt auch mein Fahrrad zu Hause. Also: Lieber sicher und unspektakulär - mit der Strassenbahn.

Rede ich zu schnell, Herr Doktor? Können Sie mir folgen? Mein Vater sagt mir seit zwei, drei Jahren immer - etwas langsamer, Marusch, ich bin weit über sechzig, bei deinem Tempo komme ich nicht mit. Das ist bei Ihnen nicht der Fall, ich weiss ... nein, nein, nach Ihrem Alter habe ich nie gefragt, das kann weiter Ihr Geheimnis bleiben; aber bitte: nicht dass mich Ihr Alter nicht interessieren würde, dass ich nicht neugierig wäre, das nicht; ich nehme nur an, ich würde einige listige Gegenfragen hören und keine brauchbare Antwort bekommen.

\section{«Rede ich zu schnell,}

\section{Herr Doktor?}

\section{Können Sie mir folgen?»}

Sorry, wo war ich jetzt stehen geblieben? Bei der Rosmarie ... nein, noch nicht, bei der Strassenbahn, richtig. Dort musste ich nämlich ein paar Minuten warten, die Nummer achtzehn ist mir direkt vor der Nase davongefahren. Ich ärgerte mich und ich wusste: Wenn man mich jetzt anquatscht, könnte ich grob werden. Zum Glück kam bald Rosmarie, meine Hausnachbarin und inzwischen auch neue Freundin. So schien es mir im ersten Augenblick; schnell war mir allerdings klar, nein, sie war es nicht, es war eine fremde und etwas ältere Frau, Rosmarie nur sehr ähnlich.

Bitte? Ja, ich verstehe, Herr Doktor. Rosmarie: Ich habe schon ein paar Mal über sie gesprochen, vor zwei Monaten etwa ... natürlich, alles kann man nicht im Kopf behalten, das begreife ich. Kurz: Nicht vielleicht die beste, aber eine gute Freundin ... wie? Nein, nein, keine Berufskollegin. Wir kennen uns vom Fitnessstudio her, sie ist inzwischen auch meine zuverlässigste Wanderpartnerin; immer fröhlich und optimistisch; sie ist möglicherweise noch Berg-fitter als ich. Am vorletzten Wochenende waren wir zum Beispiel auf dem Säntis, zum dritten Mal in diesem Sommer ... Wie? Langweilig? Ach nein, nicht die Spur, wir wählen immer eine andere Route; zum nächsten Mal aber, sagte sie, nehmen wir auch einen Mann mit, vielleicht sogar zwei, falls wir gut in Form sind. Ich war nicht dagegen, hatte allerdings meine Bedenken: $\mathrm{Ob}$ wir in den folgenden Wochen die richtigen Bergpartner finden würden? Rosmarie meinte, doch, doch ... bestimmt. Wir lachten. Am Anfang also immer die gleichen Spielchen und Sprüche. Rosmarie ist ebenfalls geschieden - ein kleiner Vorsprung: zwei Mal - ebenfalls kinderlos. Es ist uns beiden nicht ganz recht, natürlich. Der Zug ist uns noch lange nicht abgefahren, meine Liebe, sagt sie mir ab und zu; nur unbegrenzte Zeitreserven haben wir leider nicht mehr, das sei mir hoffentlich klar; wir sollten langsam Gas geben. Ja, sollen ... nicht müssen, bitte, auch darin waren wir uns schnell einig.

Ach, Rosmarie! Ich mag sie. Über die Berge sind wir uns näher gekommen, tja, vielleicht wird sie zu meiner besten Freundin avancieren, wer weiss. Eine echte Bernerin und dennoch: Nicht auf den Mund gefallen, schlagfertig, schnell und ziemlich frech. Ich wunderte mich allerdings: Ihre Männer bitte, nicht unbedingt mein Geschmack waren fast immer Machotypen mit einem dicken Schlitten und oft, traurig, aber wahr, mit einem goldenen Kettchen am Handgelenk.

$\mathrm{Na}$ ja, wir schenken uns nichts, die Rosmarie und ich, wir gehen ziemlich schonungslos miteinander um. Wie hältst du das nur aus?, fragte ich sie vor zwei Wochen nach unserem Säntisausflug; mit solchen Idioten würde ich nicht einmal einen Sonntagsspaziergang oder einen Zoobesuch un- 
ternehmen wollen, mit oder ohne anschliessendem Bettereignis. Musst du gar nicht, mein Täubchen, gab sie sofort zurück; ich würde es auch nicht fertigbringen, mit deinen Softies und penetranten Langweilern einen simplen Kinobesuch erfolgreich zu absolvieren.

Moment! Von Rosmarie wollte ich jetzt gar nicht sprechen, ich hatte mir doch Philipp vorgenommen. Zum «Mondial» also ... mit dem Tram. Ich landete dort mit einer fast halbstündigen Verspätung, es war mir nicht recht. Philipp sass an einem gedeckten Tisch - schön, da war ich eigentlich froh - lächelte, ich bekam einen Kuss auf beide Wangen. Er beteuerte, es sei schon in Ordnung, die Verspätung sei gar nicht so gross gewesen ... das «Mondial» sei übrigens eine tolle Idee gewesen. Danke!

Danke? Wofür, Philipp?, wunderte ich mich. In erster Linie müsste ich mich bei dir bedanken, dass du geduldig warst, dass du so lange - «brav» wollte ich sagen, sagte es aber nicht - auf mich gewartet hast. Oh, meinte er, bitte, bitte, nicht der Rede wert, Marusch, das war doch selbstverständlich!

Und der Abend selber? Ein wenig langweilig, am Anfang, gepflegt langweilig, sagen wir, aber im Prinzip in Ordnung.

Ähnlich übrigens wie unsere ersten zwei Treffs ... falls Sie sich, Herr Doktor, an meine damaligen Berichte noch erinnern. Philipp ist ein sehr gut informierter und - vor allem in der Aussenpolitik - belesener Mann, ich diskutiere gerne mit ihm. Es ist oft lustig und fast immer eine angenehme Horizonterweiterung. Dieses, das dritte Meeting, wird vermutlich ähnlich verlaufen, dachte ich, das heisst höflich und im Zeichen einer radikalen Keuschheit. Zu Hause aber, im Bad beim Schminken, hatte ich mir gesagt, ja, Marusch, heute bist du relativ gut drauf, da kannst du vielleicht die Zügel aus der Hand geben und dich überraschen lassen ... ja, nur so.

Was wir an jenem Abend gegessen haben, weiss ich jetzt nicht mehr ... es ist uninteressant, hoffentlich auch für Sie, Herr Doktor. Getrunken haben wir Müller-Thurgau, weiss - er, und einen lieblichen Weissherbst - ich. Er sprach viel, am Anfang über Syrien und den Nahen Osten allgemein, später über den Islam, in erster Linie über die Frau in der islamischen Welt. Ich war beeindruckt, was er alles wusste und wie locker er so ein relativ schwieriges Thema rüberbrachte. Also! Es hätte vielleicht ein schöner Abend werden können, wenn ... eben, wenn der Blumenverkäufer nicht gekommen wäre. Dunkel war er, aber kein Schwarzer, ich tippte bei seiner Herkunft vor allem auf Indien. Ein hübscher Bursche, noch jung, ziemlich gross gewachsen. Ich dachte, er

\section{«Oder haben wir noch \\ einen Drink zugute,}

\section{bei mir?»}

würde uns zeigen, was er bei sich habe, und sonst nichts sagen oder etwas Leises in rudimentärem Englisch murmeln. Falsch! Er redete eine akzeptable Dialektmischung, und zwar sehr schnell. Zuerst sprach er mich an, ich sagte klar und deutlich - nein, danke, dann wendete er sich an Philipp: Einer schönen Dame sollte man doch auch schöne Blumen schenken und diese - er hob ein wenig seine kleine Blumensammlung - sind schön ... und frisch.

$\mathrm{Na}$ ja, gerade frisch sahen seine Rosen nicht aus; das war nämlich der Grund, warum ich nein gesagt hatte und dabei auch bleiben wollte. Jetzt wendete sich der Junge an Philipp: Und die Farbe, meinte er, die passe ideal zum Foulard der Dame - beide rosa, nur in der Tönung gäbe es einen feinen Unterschied. Philipp lächelte gequält: Ja ... wenn Sie meinen. Was kostet so eine Rose?

Das hätte er nicht unbedingt fragen müssen! Der Blumenbursche nannte eine Zahl - keine Ahnung, welche - Philipp nickte und kaufte. Eine. Bitte, nur ein Exemplar! Nur ein müdes, trauriges Occasionsstück.

Gefällt sie dir?, fragte ich ihn, sei ehrlich, Philipp! Er schaute sich um, er hatte vermutlich Angst, dass der Junge uns hören könnte. Ja, warum, Marusch?, sagte er leise. Dir nicht? ... Nicht unbedingt, mein Lieber. Sie überlebt höchstens ein oder zwei Tage; ich hab ihm doch danke, nein, gesagt.

Er schaute mich traurig an. Entschuldige ... es tut mir leid ... Gut, gut! Schon passiert, Philipp. Nicht so schlimm!

Wir blieben lange im «Mondial», ich musste mich selber wundern. Wir liessen wieder Wein kommen, dann Dessert und noch einmal Wein. Die Stimmung fand ich jetzt normal, stellenweise ausgezeichnet. Etwa um halb elf zahlte er. Die Rechnung war relativ hoch, obwohl wir beide am Anfang keine Vorspeise bestellt hatten; okay, in der zweiten Hälfte wurde allerdings tüchtig gebechert, denn auch Philipp war an diesem Abend, wie sich bald herausstellte, ohne Auto gekommen.

Er rundete auf, eher minimal, um es höflich auszudrücken, ich hätte mich in seiner Haut geniert. Er offensichtlich gar nicht, denn er bat den Kellner sogar, für uns ein Taxi zu bestellen. Peinlich ... Entschuldigung, Herr Doktor, ich bin heute ziemlich weitschweifig. Na ja, Sie sind es aber gewöhnt, dass hier brutal monologisiert wird, das gehört zur Methode, ich weiss.

Ein Taxi war in knapp drei Minuten da, viel schneller, als wir gedacht hatten. Vorher kein Wort darüber, wohin die heutige Reise gehen sollte, obwohl wir schon vor der geöffneten Hintertür des Wagens standen. Willst du nach Hause fahren, Marusch?, fragte er. Oder haben wir noch einen Drink zugute, bei mir?

Haben wir?, fragte ich zurück. Nun, wenn du meinst ... ich will dir nicht wider- 
sprechen. Er freute sich. Im Auto schwiegen wir zuerst, später nahm er meine Hand und küsste sie; nur ein Hauch, kaum spürbar, dann länger und heftiger.

Nach etwa zehn Minuten waren wir am Ziel. Ein gepflegtes Vierfamilienhaus, zwei grosse Buchspflanzen in malerisch bemoosten Steintöpfen links und rechts des Eingangs. Wir stiegen aus. Und die Rose?, fragte er. Ach, vergessen! Schon vorher, im Lokal. Das tut mir leid, Philipp. Kein Problem, Marusch! Ganz frisch war sie ohnehin nicht.

Können Sie noch zuhören, Herr Doktor? Ich möchte mich nicht umdrehen - habe aber das Gefühl, dass es Ihnen heute ein wenig schwerfällt, ruhig zu sitzen. $\mathrm{Na}$ ja, vielleicht täusche ich mich. Keine Angst, jetzt rede ich nicht mehr lange ... und ich werde brav liegen bleiben, also kein Kontrollblick.

Zurück zum Taxi. Philipp bezahlte. Und wieder: Er rundete nur minimal auf und wollte noch etwas Beschwichtigendes zu unserer vergessenen Rose sagen ... in dem Moment wurden wir aber angequatscht - eigentlich mehr der Taxifahrer als wir. Ein Mann um vierzig herum, dunkler Anzug, einen hellbeigen Schal modisch um den Hals gewickelt, elegant. Allerdings nicht mehr ganz nüchtern; falsch: Ziemlich besoffen war der Mensch. Ins Zentrum, lallte er, ich meine ... zum Bahnhof, du weisst doch, wo der Bahnhof bei euch ist. Unser Fahrer zuckte die Schultern, schaute uns an und dann den Betrunkenen: Kommt nicht in Frage, rief er durch das halbgeöffnete Fenster; das Auto ist frisch geputzt ... ich will hier keine Sauerei. Adieu!

Der Elegante rastete aus ... aber total! Er lief - torkelte - dem Taxi einige Meter nach, bückte sich, vielleicht suchte er nach einem Stein, richtete sich gleich wieder auf, rannte ein Stück weiter und schrie: Du Hurensohn, du Bastard, du verdammtes Schwein! Plötzlich kehrte er um. Er ging auf uns zu und brüllte weiter: Was steht ihr da? Was gafft ihr ... was wollt ihr von mir? Haut ab! Oder nur du ... die Schlampe kannst du mir dalassen.

Philipp nahm mich am Unterarm und zog mich weg. $\mathrm{Zu}$ seinem Haus waren es knapp vierzig Meter. Wir gingen schnell. Der Elegante lief uns nicht nach, er beschäftigte sich wieder mit seiner gerade erlittenen
Niederlage und rief dem verschwundenen Taxifahrer Unschönes nach.

Das war mir vor der Tür klar: Wenn wir angegriffen worden wären, hätte ich unsere Verteidigung übernehmen müssen. Ich wartete, ob Philipp den Zwischenfall irgendwie kommentieren würde, nein, das tat er nicht; die «Schlampe» erliess er ihm. Gut, gut, seine Sache.

Die Wohnung: gross, hell, modern eingerichtet, sie interessierte mich allerdings in diesem Moment nicht besonders. Sie, Herr Doktor, vermutlich auch nicht. Lassen wir das Thema also.

Philipp hatte in seinem Keller nur Weissweine gebunkert, rote vertrage er nicht, sagte er. Komisch, ich war relativ schnell betrunken, dabei aber gar nicht in Stimmung; diese Variante kannte ich bei mir eigentlich nicht.

\section{«Was Geschmack betrifft, hätten wir sicher keine grossen Verständigungs- sorgen.»}

Küssen liess ich mich, bloss auf die Wangen, klar, auch meine Brüste durfte er zu Beginn kurz streicheln. Die ganze Gegend unter der Gürtellinie blieb jedoch tabu. Nach zwei, drei abgewehrten Versuchen kapierte er es endlich und gab auf. Wir tranken weiter, lachten viel, ausgesprochen trist war der Abend doch nicht, das will ich jetzt unterstreichen, Herr Doktor, damit kein falscher Eindruck entsteht. Fertig lustig war es erst dann - zwei leere Flaschen standen bereits auf dem Sofatischchen - als er wieder mit dem Fummeln anfing und meine Hand auf seine hungrige und zuckende Hosenwölbung legen wollte. Ich blieb höflich, sagte bloss - ich muss ... und schob seine Hand, die mein Handgelenk sanft umklammerte, langsam, aber bestimmt weg; dann verschwand ich im Bad. Ich musste tatsächlich, es war nicht gelogen, pinkeln, und zwar dringend.
Als ich zurückkam und mich hinsetzte, sah ich, dass unsere Gläser wieder voll waren. Ich bemerkte: Du bist wahrscheinlich ein besserer Trinker als Verteidiger. Ja, brummte er, ja, vermutlich ... aber wie meinst du das, Marusch? So wie ich es sage, lieber Philipp; als Verteidiger, Kavalier und Frauenbeschützer bist du grottenschlecht, ja, eine Null. Sein Blick - ziemlich gläsern verriet mir natürlich, dass auch er schon betrunken war. Ja, grottenschlecht ... ich liess nicht nach; dieser Mensch hat mich beleidigt, er hat mich Schlampe genannt.

Philipp schaute mich überrascht an: Aber ... aber, Marusch, du weisst doch, der Mann war total besoffen ... Richtig! Und? Was willst du damit sagen? ... Was hätte ich machen sollen, du hast ihn auch gesehen ... Was? Vielleicht nicht viel machen, etwas allerdings schon, mein Lieber, dachte ich.

Er seufzte laut: Ach, Marusch, komm, wir wollen jetzt nicht streiten, das führt nirgends hin; sollen wir uns den Abend durch einen betrunkenen Kerl vermiesen lassen?

Ich war unschlüssig ... und schüttelte den Kopf; vermutlich falsch. Philipp stand auf und beugte sich zu mir; er lächelte und gab mir einen Kuss ins Haar. Jetzt muss ich auch, flüsterte er und verschwand im Bad. Ich trank mein Glas zu Ende und fischte sofort das Handy aus der Tasche. Immer noch genug Saft? Ja, der Akku war geladen.

Er ging. Ich schaute mich um, die Wohnung war wirklich klasse - was Geschmack betrifft, hätten wir sicher keine grossen Verständigungssorgen.

Die Geräusche aus dem Badezimmer waren unmissverständlich: zuerst die WC-Spülung, später ein langes, monotones Summen - Philipp duschte. Okay, nichts dagegen, sein gutes Recht. Und dann noch ein Blick ins Wohnzimmer, der letzte.

Ich stand auf. Langsam packte ich meine Sachen zusammen, trank auch sein Glas leer und ging. Die grösste Sorge - das Haus könnte schon abgeschlossen sein - erwies sich als unbegründet.

Und? Ist das alles?

Mein Gott! Warum die Frage, Herr Doktor? Ja, alles!

Dr. med. AdolfJens Koemeda 